\title{
Hepatitis B Versus Hepatitis C in Blood Donors
}

\author{
Ashok Yadav*, Ravi Jain \\ Department of Pathology, MGM Medical College, Indore. India
}

\begin{abstract}
Introduction: Transmission of infectious diseases through donated blood is of concern to blood safety as transfusion forms an integral part of medical and surgical therapy. Blood transfusion carries the risk of transfusion-transmissible infections, including HIV, hepatitis, syphilis, malaria and infrequently toxoplasmosis. With every unit of blood, there is $1 \%$ chance of transfusion-associated problems including transfusion-transmitted diseases.Among all infections HIV and hepatitis are the most dreadful.

Aims \& Objectives: To find out the seroprevalence of hepatitis B virus and hepatitisC virus in blood donors, to determine the incidence of transfusion related disease in blood donors, to find the incidence of spectrum of diseases in blood bank donation, to find the age distribution of the cases studied and to find the sex distribution of the cases studied.

Material \& Methods: The present study is being undertaken in the Department of Pathology MGM Medical College Indore. This is a retrospective study that will be conducted, during the period $2008-2013$. Tests are routinely done on every blood unit to exclude HIV, HBV, $\mathrm{HCV}$, syphilis and malaria. Donors were selected by the standard criteria for donor fitness. The screening for HIV was done by ELISA using kits. HBS Ag was detected by ELISA. Anti-HCV test was done by ELISA.

Results: In the present study, 94721 blood donors are observed in the year 2008-13 in the M. Y. Blood Bank.Majority of donors are voluntary donors $76.25 \%$ as compared to replacement/relative donors $23.74 \%$.Out of total 94721 blood donations, majority of donors are male donors $94.9 \%$ (89900) as compared to female donors $5.08 \%$ (4821).The seroprevalence of HBV\&HCV is $1.83 \%$ \& $0.07 \%$ respectively. Seroprevalence is higher in the age group 26-35 year for HBV $(0.93 \%)$. HCV $(0.025 \%)$ is prevalent higher in age group $18-25$ year. Over all Seroprevalence of transfusion transmitted disease in all donations in the year 2008-13 is $1.91 \%$. Seroprevalence of transfusion transmitted disease is higher in voluntary donors $1.44 \%$ as compared to replacement/relative $0.532 \%$ donors.

Conclusion: voluntary blood donation should be encouraged for prevention of transfusion-transmissible diseases. The time and cost involved in screening donated blood can be reduced by an effective donor education and selection program that promotes self-exclusion by donors at risk of transfusion-transmissible infections. Introducing nucleic acid testing (NAT) for HBsAg and HCV is recommended to detect the infection during window period.
\end{abstract}

Keywords: Hepatitis B, Hepatitis C, Transfusion transmitted diseases, Voluntary donors, Replacement donors

\section{Introduction}

Transmission of infectious diseases through donated blood is of concern to blood safety as transfusion forms an integral part of medical and surgical therapy. Blood transfusion carries the risk of transfusion-transmissible infections, including HIV, hepatitis, syphilis, malaria and infrequently toxoplasmosis, Brucellosis and some viral infections like CMV, EBV and herpes.

With every unit of blood, there is $1 \%$ chance of transfusionassociated problems including transfusion-transmitted diseases.Among all infections HIV and hepatitis are the most dreadful. The first case of transfusion-associated AIDS was described in an infant given transfusion for erythroblastosis foetalis. There after, many cases were reported all over the world in which transfusion of blood and its products was the only risk factor.The improved screening and testing of blood donors has significantly reduced transfusion-transmitted diseases in most developed countries. This has not been so in developing nations. Poor health education and lack of awareness result in the reservoir of infections in the population.

\section{Material and Methods}

The present study is being undertaken in the Department of Pathology MGM Medical College Indore. This is a retrospective study that will be conducted, during the period $2008-2013$. Tests are routinely done on every blood unit to exclude HIV, HBV, HCV, syphilis and malaria. Donors were selected by the standard criteria for donor fitness. The screening for HIV was done by ELISA using kits. HBS Ag was detected by ELISA. Anti-HCV test was done by ELISA.

$\mathrm{ABO}$ and Rhesus (Rh) blood groups were determined using blood grouping antisera: anti-A, anti-B, anti-AB, and anti-D. Selection of cases for the study included the donors of MYH Blood Bank. 
Aim

1. To find out the seroprevalence of hepatitis B virus and hepatitisC virus in blood donors.

2. To determine the incidence of transfusion related disease in blood donors.

3. To find the incidence of spectrum of diseases in blood bank donation.

4. To find the age distribution of the cases studied.

5. To find the sex distribution of the cases studied.

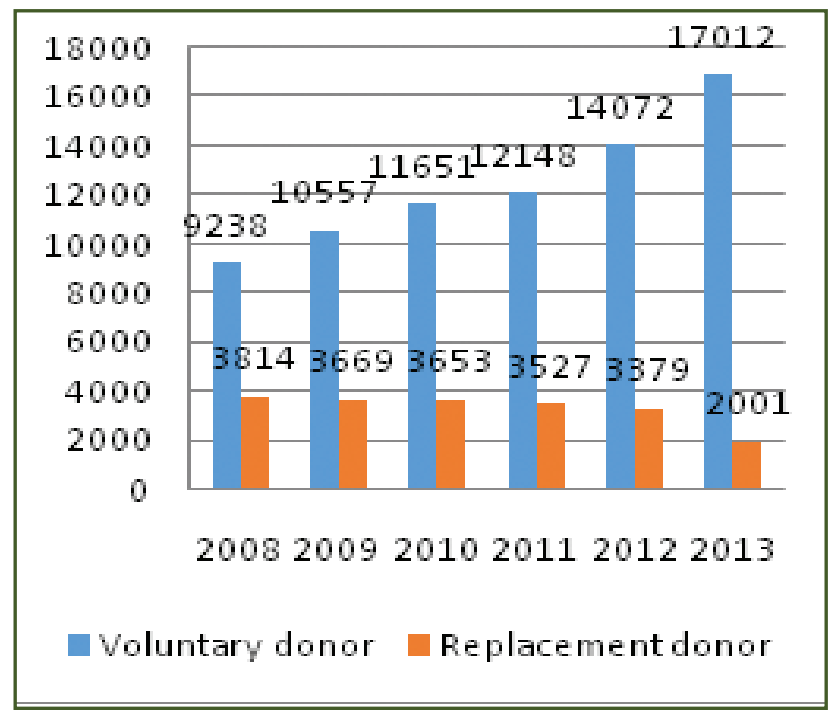

Graph 1: Number of blood units collected during the year 2008-13. Out of total 94721 blood donations, majority of donors are voluntary donors $78.83 \%$ as compared to replacement/relative donors $21.17 \%$

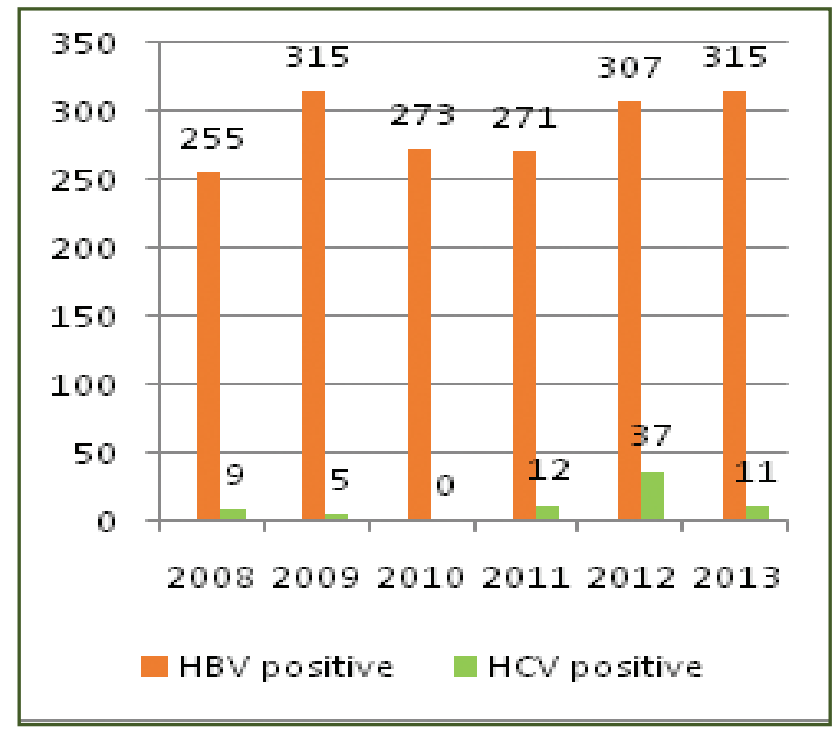

Graph 3 : Seropositive donors for HBV and HCV in 200813 Seroprevalence of HBV and HCV are $1.83 \%$ and 0.07 $\%$ respectively.

Annals of Applied Bio-Sciences, Vol. 4; Issue 1: 2017

\section{Results}

The present study is conducted in the Department of Pathology MGM Medical College Indore and M. Y. Hospital blood bank. This is a retrospective study that was conducted, during the period 2008 -2013. In the present study, 94721 blood donors are observed in the year 200813 in the M. Y. Blood Bank. The data collected from donor register record book, donors form, master record book, $\mathrm{HIV}, \mathrm{HBV}$ and HCV positive beg number records. The results and observations studies are presented below:

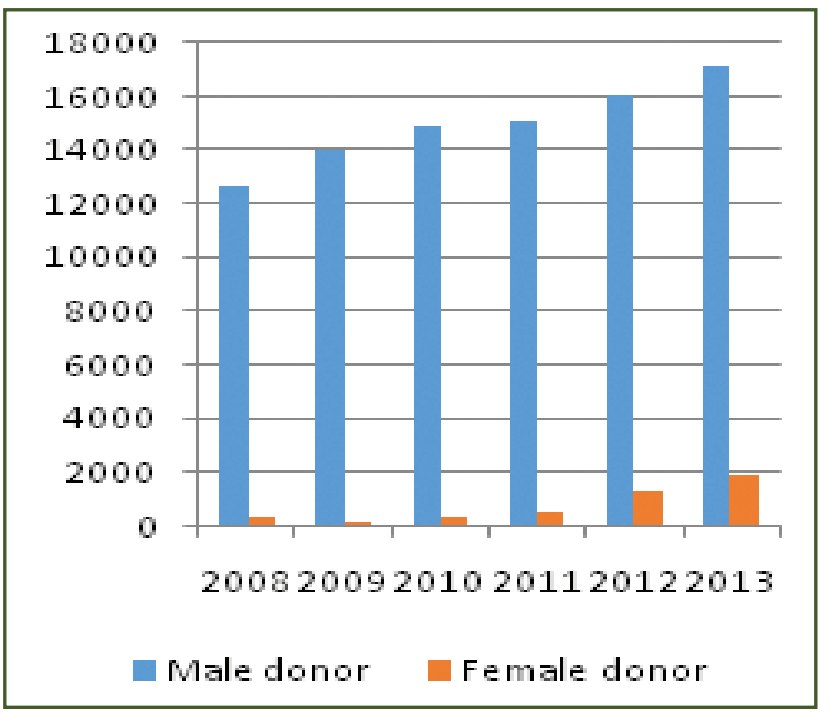

Graph 2 : Number of male and female donors during the year 2008-13.0ut of total 94721blood donations, majority of donors are male donors $94.9 \%$ as compared to female donors $\mathbf{5 . 0 8 \%}$

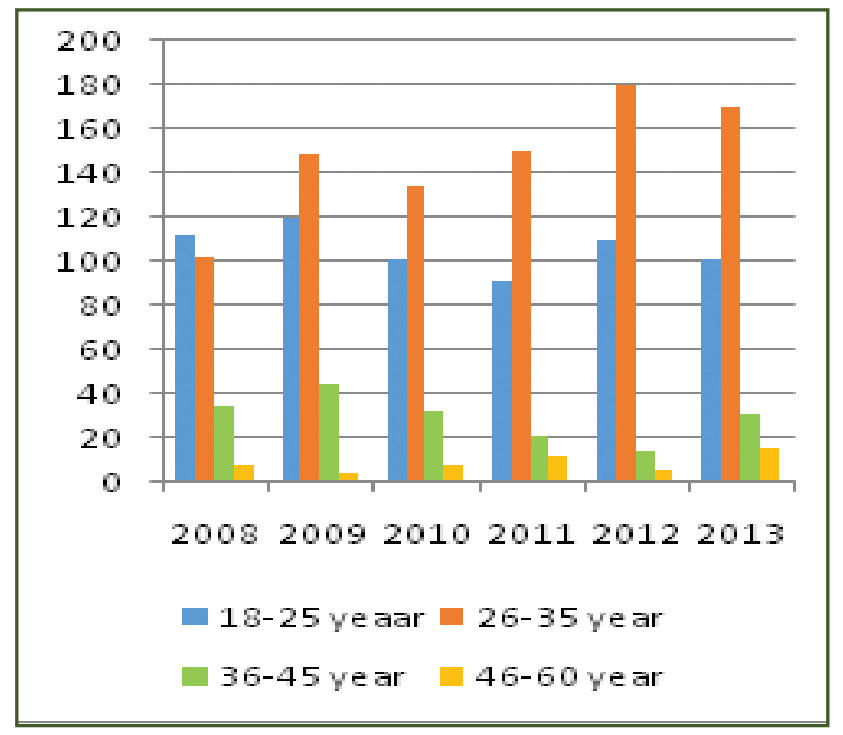

Graph 4: Age wise distribution of HBV in the year 200813. Seroprevalence is higher in the age group 26-35 year 


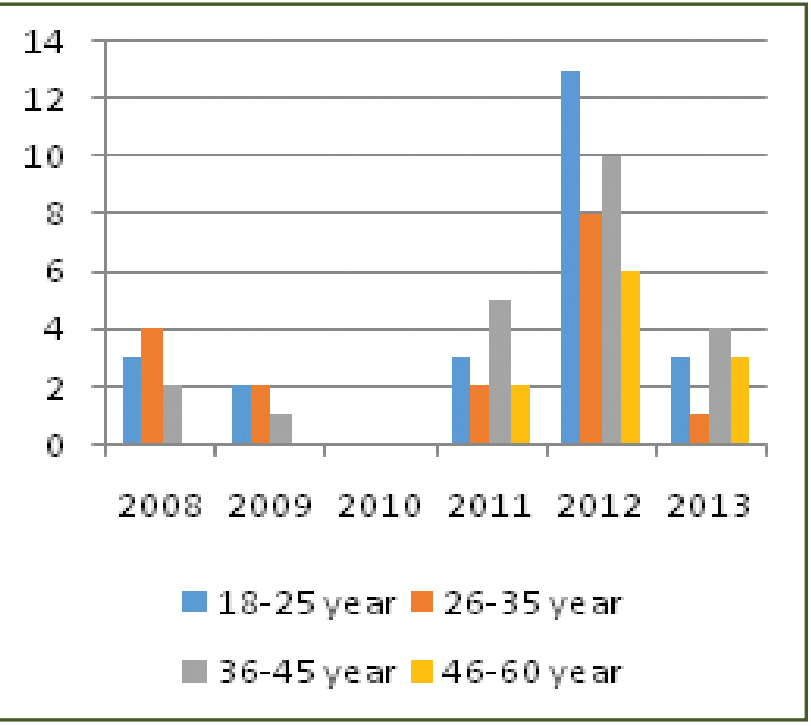

Graph 5 : Age wise distribution of seroprevalence of HCV in the year 2008-13. Seroprevalence is higher in age group 18-25 yrs in year 2011,12\& 2013.

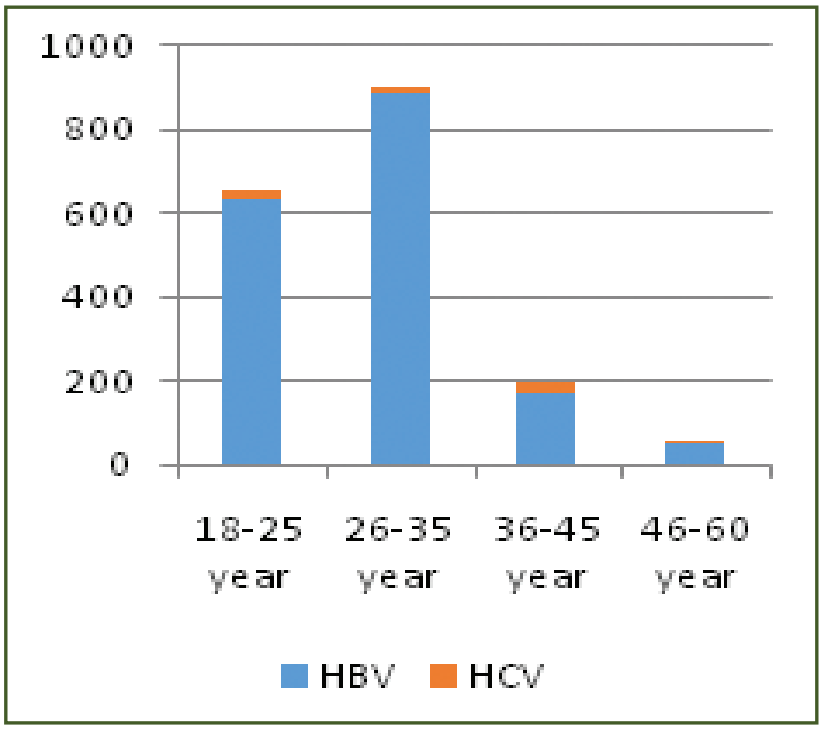

Graph 6: Overall age distribution of seroprevalence of $\mathrm{HBV}$ and $\mathrm{HCV}$ in 2008-2013. HBV is more prevalent in age group 26-35 years while $\mathrm{HCV}$ is more prevalent in $\mathbf{1 8 - 2 5}$ years.

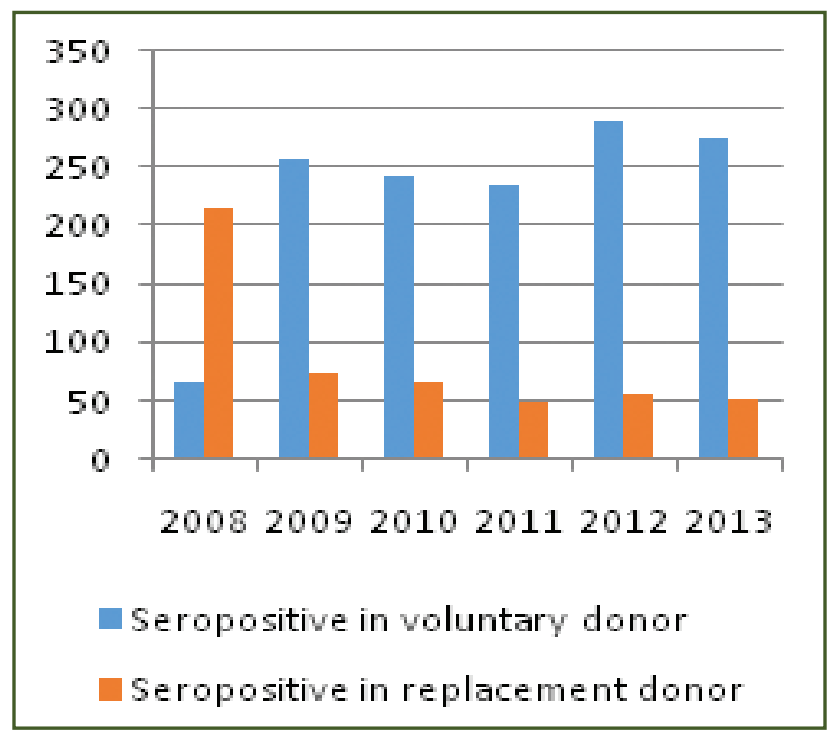

Graph 7 :Seropositivity of transfusion transmitted disease in 2008-13.Seropositivity is higher in Voluntary donors in all years except year 2008 where seropositivity is higher in replacement donors.

\section{Discussion}

There are four main groups of micro-organisms known to cause infections namely viruses, bacteria, protozoa and fungi. Only first three groups of microbes - viruses, bacteria and protozoa - have been reported to be transmitted by blood transfusion. Individuals with fungal infections are usually too sick to be accepted as blood donors. Viruses are most commonly transmitted by transfusion.
Recently, a new form of infectious agent - the prion - has been identified. At this time, there is no evidence to suggest that they could be transmitted by blood transfusion.

Viruses are the simplest forms of life. They infect all forms of life, they lack certain components needed to live and their growth hence depend on the host cell that they infect to provide these missing components.Following are some of the viruses which are known to be transmitted through blood: 
1. Human immunodeficiency virus (HIV)

2. Hepatitis B virus

3. Hepatitis $\mathrm{C}$ virus

4. Hepatitis A virus

5. Hepatitis $G$ virus

6. Non - A, Non - B Hepatitis

7. Epstein Barr Virus

8. Cytomegalo virus (CMV)

9. Human T Lymphocytic virus (HTLV - 1 \& HTLV - 2)

Hepatitis $\mathbf{B}$ is an infectious inflammatory illness of the liver caused bythe hepatitis B virus (HBV) that affects hominoidea, including humans. Originally known as "serum hepatitis", 2 the disease has caused epidemics in parts of Asia and Africa, and it is endemic in China. ${ }^{3}$ About a third of the world population has been infected at one point in their lives, ${ }^{4}$ including 350 million who are chronic carriers. ${ }^{5}$

The virus is transmitted by exposure to infectious blood or body fluids such as semen and vaginal fluids, while viral DNA has been detected in the saliva, tears, and urine of chronic carriers. Perinatal infection is a major route of infection in endemic (mainly developing) countries. ${ }^{6}$ Other risk factors for developing HBV infection include working in a healthcare setting, transfusions, dialysis, acupuncture, tattooing, extended overseas travel, and residence in an institution. ${ }^{4,7}$ However, Hepatitis B viruses cannot be spread by holding hands, sharing eating utensils or drinking glasses, kissing, hugging, coughing, sneezing, or breastfeeding. ${ }^{4}$

The transmission of HBV is mainly by parental route which involves direct contact with body fluid. The most common route of infection are:

- Contact with infected blood, either by exposure of wounds to infected blood or to contaminated needles and syringes used in injecting drugs, tattooing, ear piercing, or acupuncture.

- Sexual contact

- Neonatal or perinatal transmission

- Transfusion of infected blood or blood products. ${ }^{1}$

Hepatitis $\mathbf{C}$ is an infectious disease affecting primarily the liver, caused by the hepatitis $\mathrm{C}$ virus (HCV). ${ }^{[8]}$ The infection is often asymptomatic, but chronic infection can lead to scarring of the liver and ultimately to cirrhosis, which is generally apparent after many years. In some cases, those with cirrhosis will go on to develop liver failure, liver cancer or life-threatening esophageal and gastric varices. ${ }^{9}$

$\mathrm{HCV}$ is spread primarily by blood-to-blood contact associated with intravenous drug use, poorly sterilized medical equipment and transfusions. Hepatitis $\mathrm{C}$ only infects humans and chimpanzees. ${ }^{10} \mathrm{Hepatitis} \mathrm{C}$ is the leading cause of liver transplantation, though the virus usually recurs after transplantation. ${ }^{11}$ No vaccine against hepatitis $\mathrm{C}$ is available.

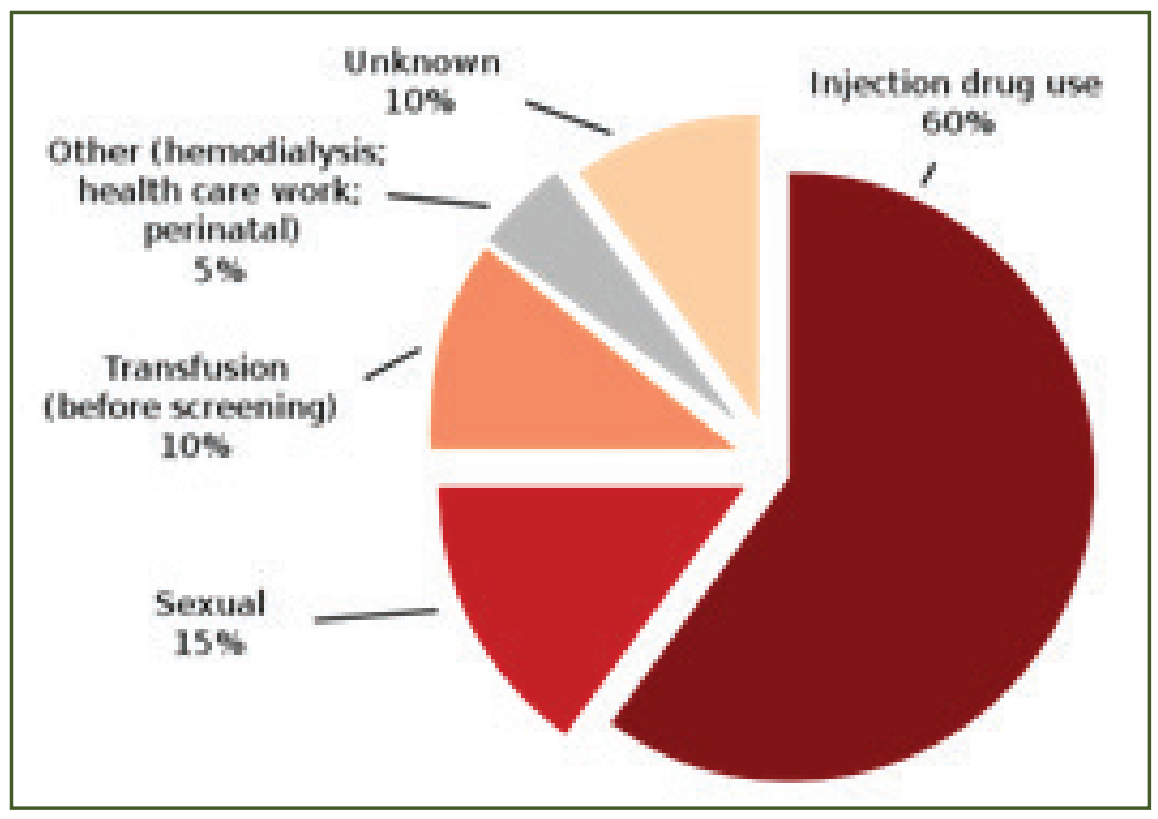

Figure 1: Transmission 
Voluntary or Replacement/Relative Donor -In our study, out of total 94721 blood donations, majority of donors are voluntary donors $76.25 \%$ as compared to replacement/ relative donors $23.74 \%$. Similarly majority of donors are voluntary in another study out of 19135 blood donors, 11165 (58\%) were voluntary and 7970 (42\%) were replacement/ relative donors by Nagarekha Kulkarni ${ }^{12}$ Associate Professor, Department of Pathology, Vijayanagara Institute of Medical Sciences, Bellary - 583104, Karnataka, India.

Male or Female Donor: In our study, out of total 94721 blood donations, majority of donors are male donors 94.9 $\%$ (89900) as compared to female donors $5.08 \%$ (4821). Similarly another study is comparable for majority of donors are male $96.22 \%$ by Dimple Aroraand Bharti Aroraet $\mathrm{al}^{13}$ in Haryana. In the another study, the percentage of male patients was $73 \%(860 / 1178)$ as compared with $27 \%$ (318/1178) for female patients by Manisha Jain and Anita Chakravarti et $\mathrm{al}^{14}$. conducted in New Delhi.

Seroprevalence of HBV: In our study, the seroprevalence of $\mathrm{HBV}$ is $1.83 \%$ in total blood donations in the year 2008-13. Seroprevalence of HBV is comparable to another study with seroprevalence of HBS Ag was 1.7 $\%$ by Dimple Aroraand Bharti Aroraet al ${ }^{13}$ conducted in Haryana.The seroprevalence of hepatitis B surface antigen was $0.87 \%$ noted in hospital-based population by SmitaSood and ShirishMalvankar et al ${ }^{15}$ conducted in Rajasthan.In another study conducted among donors of interior Sindh (Pakistan) by Mujeeb et $\mathrm{al}^{18}$, the seroprevalence of $\mathrm{HBV}$ was $6.2 \%$.

Seroprevalence of HCV: In our study, the seroprevalence of HCV is $0.07 \%$ in total blood donations in the year 200813.It is comparable to another study, theseroprevalence of HCV $0.072 \%$ by Murphy EL, Fang J,Tu Y, et al. conducted in USA. This seroprevalence is much lower than the 0.4$5.2 \%$ seroprevalence reported in an earlier study conducted in various European countries by Hahneet al ${ }^{17}$. Another study by Viet Le at $\mathrm{al}^{19}$ found HCV prevalence to be $0.17 \%$.

Age wise distribution: In our study, Seroprevalence is higher in the age group 26-35 year for HBV (0.93\%). $\operatorname{HCV}(0.025 \%)$ is prevalent higher in age group 18-25 year .In another study,the highest seroprevalence of anti$\mathrm{HCV}$ was found in males above the age of 61 years. The highest seroprevalence for anti-HIV was found in the age group 31-40 years by SmitaSood and ShirishMalvankar et $\mathrm{al}^{15}$ conducted in Rajasthan.In another study, 3 positive cases belonged to 21-40 years age group by S Mishra and $\mathrm{N}$ Chayani et $\mathrm{al}^{16}$ conducted in Orissa.

\section{Summary and Conclusion}

The present study is conducted in the Department of Pathology MGM Medical College Indore and M. Y.
Hospital blood bank. This is a retrospective study that was conducted, during the period $2008-2013$. Tests are routinely done on every blood unit to exclude HIV, HBV and HCV. Donors were selected by the standard criteria for donor fitness. The data collected from donor register record book, donors form, master record book, HIV, HBV and HCV positive beg number records. Out of total 94721 blood donations, majority of donors are voluntary 76.25 $\%$ as compared to replacement/relative donors 23.74 $\%$.Seroprevalence of $\mathrm{HBV}$ and $\mathrm{HCV}$ are $1.83 \%$ and $0.078 \%$ respectively. Seroprevalence of HBV is higher than HCV.Seroprevalence is higher in the age group 2635 year for HBV-0.904\%. For HCV-0.025\%, it is higher in age group 18-25 yearsAge group 26- 35 year are show higher Seroprevalence $(0.952 \%)$ for HBV \& HCV.. Over all Seroprevalence of transfusion transmitted disease in all donations in the year 2008-13 is $1.91 \%$. Seroprevalence of transfusion transmitted disease is higher in voluntary donors $1.44 \%$ as compared to replacement/relative 0.532 $\%$ donors.HBV and HIV are the most prevalent transfusiontransmissible diseases among blood donors in Indore. Screening and better selection of donors are necessary to improve blood safety in the regional blood transfusion centre of M. Y. Hospital. Therefore, it is concluded that voluntary blood donation should be encouraged for prevention of transfusion-transmissible diseases. The time and cost involved in screening donated blood can be reduced by an effective donor education and selection program that promotes self-exclusion by donors at risk of transfusion-transmissible infections. Introducing nucleic acid testing (NAT) for HBsAg and HCV is recommended to detect the infection during window period.

\section{References}

1. Tang, J. and Kaslow, R. A. (2003). "The impact of host genetics on HIV infection and disease progression in the era of highly active antiretroviral therapy". AIDS 17 (Suppl 4): S51-S60.

2. Arthos J, Cicala C, Martinelli E, Macleod K, Van Ryk D, Wei D, Xiao Z, Veenstra TD, Conrad TP, Lempicki RA, McLaughlin S, Pascuccio M, Gopaul R, McNally J, Cruz CC, Censoplano N, Chung E, Reitano KN, Kottilil S, Goode DJ, Fauci AS. (2008). "HIV-1 envelope protein binds to and signals through integrin alpha(4)beta(7), the gut mucosal homing receptor for peripheral T cells". Nature Immunol. In Press (3): 301-9.

3. Various (2008) (PDF). HIV Sequence Compendium 2008 Introduction. Retrieved 2009-03-31.

4. Haedicke, J.; Brown, C.; Naghavi, H. (Aug 2009). "The brain-specific factor FEZ1 is a determinant of neuronal susceptibility to HIV-1 infection". Proceedings of the National Academy of Sciences 106 (33): 14040-14045.

5. Arauz-Ruiz P, Norder H, Robertson BH, Magnius LO (August 2002). "Genotype H: a new Amerindian genotype 
of hepatitis B virus revealed in Central America". J. Gen. Virol. 83 (Pt 8): 2059-73.

6. Coffin CS, Mulrooney-Cousins PM, van Marle G, Roberts JP, Michalak TI, Terrault NA (April 2011). "Hepatitis B virus (HBV) quasispecies in hepatic and extrahepatic viral reservoirs in liver transplant recipients on prophylactic therapy". Liver Transpl 17 (8): 955-62

7. Schwartz O, Maréchal V, Le Gall S, Lemonnier F, Heard JM (March 1996). "Endocytosis of major histocompatibility complex class I molecules is induced by the HIV-1 Nef protein". Nat. Med. 2 (3): 338-42.

8. Alpert E, Isselbacher KJ, Schur PH (July 1971). "The pathogenesis of arthritis associated with viral hepatitis. Complement-component studies". The New England Journal of Medicine 285(4): 1859.

9. Pungpapong S, Kim WR, Poterucha JJ (2007). "Natural History of Hepatitis B Virus Infection: an Update for Clinicians". Mayo Clinic Proceedings 82 (8): 967-975.

10. Schaefer S (January 2007). "Hepatitis B virus taxonomy and hepatitis B virus genotypes". World Journal of Gastroenterology : WJG 13 (1): 14-21.

11. Pope M, Haase A (2003). "Transmission, acute HIV-1 infection and the quest for strategies to prevent infection". Nat Med 9 (7): 847-52.

12. Nagarekha Kulkarni "Analysis of the seroprevalence of HIV, HBsAg, HCV and syphilitic infections detected in the pretranfusion blood: A short report" http://www.ijbti.com/ archive/2012-archive/100006IJBTINK2012-kulkarni/index.php
13. Dimple Arora, Bharti Arora, Anshul Khetarpal "Seroprevalence of HIV, HBV, HCV and syphilis in blood donors in Southern Haryana" Year 2010 Vol: 53(2) Page308-309

14. Manisha Jain, Anita Chakravarti, VikasVerma, PreenaBhalla"Seroprevalence of hepatitis viruses in patients infected with the human immunodeficiency virus" Year : 2009 Vol: 52( 1)Page : 17-19

15. SmitaSood and ShirishMalvankar "Seroprevalence of Hepatitis B Surface Antigen, Antibodies to the Hepatitis C Virus, and Human Immunodeficiency Virus in a HospitalBased Population in Jaipur, Rajasthan"Year : 2010 , Vol : 35 (1)Page : 165-169

16. S Mishra, N Chayani, G Sarangi, B Mallick, SB Pati "Seroprevalence of anti HCV antibody in and around Cuttack, Orissa"year : 2002 Vol : 20 (1) Page : 40-41

17. Hahné SJ, Veldhuijzen IK, Wiessing L, Lim T-A, Salminen $M$, Laar $M$ van de. Infection with hepatitis $B$ and $C$ virus in Europe: a systematic review of prevalence and costeffectiveness of screening. BMC Infectious Diseases. 2013;13:181. doi:10.1186/1471-2334-13-181.

18. Mujeeb SA, Pearce MS. Temporal trends in hepatitis $\mathrm{B}$ and $\mathrm{C}$ infection in family blood donors from interior Sindh, Pakistan. BMC Infectious Diseases. 2008;8:43. doi:10.1186/1471-2334-8-43.

19. Viet L, Lan NTN, Ty PX, et al. Prevalence of hepatitis B \& hepatitis $\mathrm{C}$ virus infections in potential blood donors in rural Vietnam. The Indian Journal of Medical Research. 2012;136(1):74-81.
*Corresponding author:

Dr Ashok Yadav, Yadav clinic, New panchsheel colony, Musakhedi, Indore, India Phone: +919893273236

Email: drashokmyh@gmail.com, ravijainpatho@gmail.com

Financial or other Competing Interests: None.
Date of Submission : 04.02.2017

Date of Acceptance : 10.02.2017 Date of Publication : 15.02.2017 